\title{
Entrelacs
}

Cinéma et audiovisuel

Hors-série $n^{\circ} 4$ | 2016

Paysages en séries

\section{Le Paysage, la roche et le fer : que dit le décor d'Excalibur?}

Le paysage, la roche et le fer :

\section{Justine Breton}

\section{(2) OpenEdition}

Journals

\section{Édition électronique}

URL : http://journals.openedition.org/entrelacs/2139

DOI : 10.4000/entrelacs.2139

ISBN : 2261 - 5482

ISSN : 2261-5482

Éditeur

Éditions Téraèdre

Édition imprimée

Date de publication : 1 novembre 2016

ISSN : 1266-7188

\section{Référence électronique}

Justine Breton, «Le Paysage, la roche et le fer : que dit le décor d'Excalibur? », Entrelacs [En ligne], Hors-série n 4 | 2016, mis en ligne le 06 février 2018, consulté le 03 mai 2019. URL : http:// journals.openedition.org/entrelacs/2139; DOI : 10.4000/entrelacs.2139

Ce document a été généré automatiquement le 3 mai 2019.

Tous droits réservés 


\section{Le Paysage, la roche et le fer : que dit le décor d'Excalibur?}

Le paysage, la roche et le fer:

Justine Breton

1 La légende du roi Arthur, largement exploitée par le cinéma comme par la télévision, est marquée par quelques éléments graphiques forts, qui condensent les enjeux du mythe. Le cinéma et les séries télévisées, notamment, se plaisent à exploiter le pouvoir traditionnel et la puissance symbolique de l'épée Excalibur, plantée dans un rocher ou dans une enclume. Cette image, aisément identifiable par le spectateur, possède une existence propre dans le panorama télévisuel, et ne se limite pas au seul cadre arthurien. Cette étude propose de comparer les différents décors choisis pour la représentation d'une même scène - l'apparition d'Excalibur plantée dans la pierre - dans plusieurs séries, qui s'appuient sur une même syntaxe iconographique. En effet, l'épée n'a pas uniquement de sens en soi, mais entre également en résonnance avec le paysage dans lequel elle est présentée : à la télévision plus encore qu'au cinéma, le décor contribue à la narration, et permet d'éclairer le pouvoir associé à Excalibur.

2 Une distinction apparait entre les séries consacrées au roi Arthur et les séries nonarthuriennes, i.e. les séries télévisées qui ne prennent pas pour cadre la légende médiévale, mais qui proposent un autre type de narration dans lequel l'épée du roi ne constitue qu'une référence ponctuelle. Cette apparition d'Excalibur ne s'avère pas nécessairement décisive dans la structure de l'épisode concerné, mais permet chaque fois de souligner la légitimité, avérée ou non, du personnage mis en scène. Un décalage symbolique majeur se fait jour lorsqu'Excalibur, plantée dans un rocher - ou plus rarement dans une enclume -, est présentée dans un décor naturel, ou au contraire lorsqu'elle apparait dans un lieu clos et artificiel. Dans quelle mesure le paysage est-il valorisé pour annoncer le pouvoir conféré au possesseur d'Excalibur? Plus qu'un simple élément illustratif, le décor qui entoure l'épée devient vecteur de sens, et éclaire la lecture de la prise de pouvoir du roi Arthur. Ainsi, un paysage vaste et ouvert révèle une communion des éléments naturels autour de la figure du roi, tandis que la dissimulation de l'épée dans un espace souterrain et sombre traduit une velléité de pouvoir illégitime. 
Cette interprétation d'Excalibur par le décor s'avère essentielle dans le cadre sériel, nécessairement plus limité - au moins en durée, si ce n'est en budget - que le cinéma : outre les films arthuriens, peu de productions cinématographiques mettent en scène l'épée médiévale ${ }^{1}$.

\section{Excalibur ou la force des éléments}

3 La présentation de l'épée dans les séries arthuriennes se caractérise par son intégration à un cadre naturel où le paysage occupe une place majeure à l'écran. Excalibur apparaît alors dans des scènes extérieures où la nature joue un rôle symbolique dans la détermination du pouvoir du héros.

\section{Le rôle prépondérant du paysage naturel}

Le lien entre nature et pouvoir légitime est principalement développé dans des séries qui mettent en scène l'extraction de l'épée par le roi Arthur - ou par une figure proche de souverain légitime -, parfois à travers des représentations diffractées: Kaamelott (M6, 2005-2010), Merlin (BBC One, 2008-2012), Camelot (Starz, 2011), Once Upon a Time (ABC, 2011- ) et Galavant (ABC, 2015-2016). Selon la tradition médiévale, développée par des auteurs tels que Robert de Boron et Thomas Malory, c'est en parvenant à extraire une épée merveilleuse d'une enclume elle-même ancrée dans un perron que le jeune Arthur devient roi d'Angleterre. Ce n'est que par la suite que cette épée est associée au nom d'Excalibur, qui correspond à l'origine à une autre arme du roi. L'imaginaire collectif, notamment perceptible dans les séries télévisées, tend toutefois à identifier l'épée dans la pierre comme étant Excalibur, l'épée légendaire d'Arthur. Dans la série française Kaamelott, l'accès d'Arthur à cette arme est répété, puisque le personnage l'extrait et la plante dans la pierre à plusieurs reprises, remettant ainsi en question son autorité royale. $\mathrm{Au}$ cours de son développement, Kaamelott propose une légère évolution dans le motif d'Excalibur: dans l'une des séquences pilotes, visible dans le générique provisoire de la série, l'épée apparaît d'abord plantée de façon verticale dans un rocher, lui-même situé dans un lac. Cette localisation sert alors ponctuellement la narration : lorsqu'il parvient à extraire l'épée de la roche, Arthur, entrainé par son élan, perd l'équilibre et tombe en arrière, dans l'eau du lac. Ce gag désacralise à la fois le roi et son épée légendaire, soulignant ainsi la tonalité burlesque de la série. Cette mise en scène n'est pourtant pas conservée dans la narration finale de Kaamelott. En effet, les épisodes pilotes sont accessibles au public par les bonus de l'édition DVD, mais ne constituent qu'un reflet des balbutiements de la série: distribution non définitive, décors et accessoires encore limités, etc. La narration repart à zéro dans la première saison, permettant ainsi de proposer une nouvelle version de l'extraction d'Excalibur par Arthur (S05E12, «La Roche et le Fer »). Le paysage privilégié dans cette représentation alternative varie quelque peu : l'épée est plantée dans un angle diagonal dans un large rocher, légèrement en hauteur par rapport au roi. Cette pierre aux propriétés merveilleuses apparaît cette fois au milieu d'une plaine rocheuse, avec à proximité une forêt. Ce décor soumis à un vent froid se caractérise également par la présence fréquente de neige, dont la blancheur permet d'établir un contraste avec la noirceur de la roche ${ }^{2}$. La série Merlin reprend en partie ces éléments naturels : Excalibur y apparait plantée à la verticale dans un rocher, lequel est situé bien en évidence au milieu d'une clairière ménagée dans la forêt (S03E13, «The 
Coming of Arhur, Part $2 »)$. Il s'agit de l'une des rares occurrences audiovisuelles où est représentée en amont l'insertion de l'épée dans la pierre, bien avant qu'Arthur puisse y accéder. Merlin et Kaamelott proposent ainsi une vision diffractée d'Excalibur dans le rocher, qui apparaît à plusieurs reprises dans la narration.

Dans une perspective proche, la série Camelot met en scène deux épées destinées au jeune roi Arthur, en écho aux premiers textes médiévaux. En effet, Camelot présente d'abord l'épée de Mars, arme issue d'une légende romaine selon laquelle celui qui pourra arracher l'arme, plantée dans la roche en haut d'une chute d'eau, sera roi (S01E02, « The Sword and the Crown »). L'épée employée pour l'élection du souverain est associée à la puissance du dieu romain de la guerre, et souligne ainsi le pouvoir guerrier d'Arthur, seul héros apte à s'emparer de l'épée de Mars. La représentation de l'arme suit les codes traditionnels d'Excalibur, tant dans sa symbolique que dans les éléments du cadre : la lame est enfoncée dans la pierre, entourée d'eau vive et à proximité d'une forêt. L'épée nommée Excalibur est intégrée dans un second temps dans la série, où Merlin la récupère, brandie hors des eaux, de la main d'une jeune fille enfermée sous la surface gelée d'un lac (S01E04, « Lady of the Lake ») : si le rocher est absent de cette interprétation, la dureté de la glace propose toutefois un renvoi à l'image de la pierre.

6 Si l'épée apparaît à deux reprises dans son rocher dans Once Upon a Time, seule la première mise en scène concerne le personnage du roi. Après le générique du premier épisode de la cinquième saison, un plan d'ensemble aérien sur trois cavaliers laisse découvrir un décor de montagne ensoleillé et empli de verdure (S05E01, "The Dark Swan »). La structuration de l'image en trois bandes horizontales, composées de haut en bas par le ciel, la montagne et la végétation, insiste sur le calme de la scène et valorise le paysage. L'élargissement de l'image par les lignes de force horizontales tend à unir les éléments plutôt qu'à les séparer : le petit groupe de cavaliers chevauchant paisiblement entre terre et ciel suggère l'union des aspects matériels et spirituels dans un même dynamisme. Le mouvement latéral de la caméra suit le chevauchement des trois personnages, qui mettent pied à terre près d'une étendue d'eau. Ils avancent alors dans la forêt, sans leurs montures, et accèdent à l'épée, plantée verticalement dans un rocher placé en évidence entre les arbres. L'apparition d'Excalibur est ainsi mise en attente, et suit sa découverte par les personnages - le roi Arthur et deux de ses chevaliers. Enfin, la série Galavant, sans mettre en scène Excalibur à proprement parler, présente toutefois une épée plantée dans une souche, que seul le véritable souverain peut retirer (S02E02, "World's Best Kiss »). Le bois se substitue ici à la pierre comme support de l'épée, reprenant en écho les mythes nordiques similaires à celui d'Excalibur.

Chaque fois, les représentations sérielles proposent des variations à partir des mêmes éléments naturels. Le rocher, qui constitue un élément inhérent au prodige de l'épée, apparait parfois seul, comme dans Merlin, où il est mis en valeur par un rayon de lumière. Pour d'autres séries, la pierre est intégrée à un espace plus large, à l'instar de la cascade dans Camelot. Cette élévation de l'épée, plantée en haut d'une chute d'eau, permet de souligner le rôle essentiel accordé dans ces scènes à l'eau sous toutes ses formes: l'élément aquatique peut représenter un cadre paisible, comme dans Once Upon a Time, ou constituer un obstacle à l'extraction de l'épée, comme dans Kaamelott et Camelot, où la neige et la glace ralentissent la progression des personnages. À l'inverse, la forêt, pourtant présente de façon constante, apparaît comme un élément de décor peu exploité par les scénaristes. L'épaisseur du bois permet le plus souvent de dissimuler Excalibur avant sa révélation aux yeux de tous. Par la suite, la présence de la forêt insiste sur 
l'intégration de l'épée et du héros à un cadre pur : dans la pensée médiévale, qui ressurgit ici, la forêt constitue un lieu sauvage et naturel, en opposition avec la civilisation, perçue comme corruptrice. En faisant apparaître Excalibur en extérieur, les réalisateurs suggèrent l'absence d'artificialité dans le prodige de l'épée, lequel est présenté comme un signe divin de désignation du souverain.

Cette représentation du paysage naturel est largement accentuée par rapport aux hypotextes médiévaux, et notamment par rapport au Merlin de Robert de Boron, qui décrit l'apparition de l'épée dans une enclume devant une église. Par ailleurs, il est à noter que les séries télévisées, à l'exception de The Simpsons, privilégient la mise en scène de l'épée dans la pierre, et non dans une enclume, comme le suggérait déjà Robert de Boron (Robert de Boron, 268-269). L'image d'Excalibur dans l'enclume a elle-même été popularisée, dès 1963, par le dessin animé The Sword in the Stone de Disney. Pourtant, la télévision insiste sur l'enserrement de l'épée dans un rocher apparemment non taillé, soulignant ainsi l'intégration du prodige merveilleux dans un élément naturel. Il est possible de voir dans ce détournement l'influence du titre du célèbre roman de T.H. White, The Sword in the Stone, premier volet de son œuvre The Once and Future King et source d'inspiration de l'adaptation cinématographique par les studios Disney. En effet, si White décrit bien dans son texte une épée plantée dans une enclume, le titre popularise toutefois l'expression "l'épée dans le rocher", élément plus naturel que l'enclume (White, 200). Par ailleurs, la ligne verticale marquée par Excalibur traduit une certaine rigidité et fixe le regard du spectateur, tandis que la pierre dans laquelle elle est ancrée établit un contraste en intégrant une certaine rondeur dans l'image : la force symbolisée par l'épée ne brise pas l'harmonie de l'image, mais prolonge la présence massive et naturelle de la roche en l'étirant vers le haut.

9 L'image popularisée par la production audiovisuelle s'appuie plus aisément sur l'interprétation proposée par T.H. White que sur les textes médiévaux, bien souvent méconnus des créateurs et scénaristes. Selon Robert de Boron, l'épée permettant à Arthur d'accéder au trône apparaît avec l'enclume au milieu d'un perron, devant une église, où tous sont réunis pour célébrer la Nativité. Cet emplacement annonce le rôle prépondérant joué par les évêques dans la découverte de l'épée et dans la prise en compte de ce prodige à la fois religieux et politique. Or, par un phénomène général de sécularisation des sociétés occidentales et par la volonté des auteurs de s'approprier la légende arthurienne, le cadre urbain et chrétien d'Excalibur est délaissé dès le $\mathrm{XX}^{\mathrm{e}}$ siècle et l'épée se trouve rapidement associée à un paysage naturel. En ce sens, T.H. White permet une transition narrative, en décrivant dans The Sword in the Stone l'apparition de l'épée devant une église, mais en effaçant toute implication chrétienne au profit d'une valorisation des animaux et des éléments naturels. Dès lors, Excalibur semble automatiquement associée à la nature.

\section{L'harmonie des éléments naturels au service d'un pouvoir légitime}

10 La juxtaposition de la roche, de l'eau et de la végétation lors de l'apparition d'Excalibur permet de suggérer une forme d'harmonie entre les éléments : le pouvoir d'Arthur, lié à l'épée, est ainsi ancré dans les forces telluriques qui composent son royaume. L'épée est conçue comme un outil servant à concentrer cette puissance naturelle et devient le symbole de l'harmonie créée sous le règne naissant d'Arthur. La représentation conjointe du rocher, de l'eau et de la forêt entourant l'épée met ainsi en évidence la légitimité du roi, seul apte à s'emparer de cette arme issue de forces naturelles. La série Kaamelott 
propose justement un détournement burlesque de cette association entre Excalibur et les éléments: les proches du roi questionnent les effets néfastes que l'érosion ou les conditions météorologiques pourraient avoir sur le pouvoir de l'épée (S05E22, «La Promesse »). Ce dialogue entre la reine Guenièvre et ses parents, inquiets de voir Arthur perdre son pouvoir s'il ne parvenait pas à extraire l'épée du rocher, souligne les conséquences triviales d'une harmonie entre Excalibur et la nature :

Guenièvre: Vous pourriez peut-être faire confiance à Excalibur pour rester fidèle à son propriétaire.

Séli : On n'a pas tellement le choix... Seulement quand on prend conscience que tout tient au bon vouloir d'un bout de ferraille, on miquette un peu, c'est naturel.

Léodagan: Imaginez qu'il soit détraqué, le machin. Ah bah c'est vrai on sait pas ça: le froid, l'humidité... (S05E12, « La Roche et le Fer »)

Or, Excalibur semble directement émaner d'une puissance naturelle et n'est donc pas soumise à ces considérations matérielles. La représentation de l'épée dans des scènes extérieures permet de la décrire comme un attribut de la légitimité de la puissance du roi Arthur. Son pouvoir, symbolisé par l'épée, n'est pas dissimulé ni conçu de façon artificielle, mais est laissé à la vue de tous. Excalibur, avant de revenir au souverain, est en effet accessible à tous ceux qui souhaitent tenter leur chance, et essayer de s'emparer du pouvoir. Ce prodige, ancré dans un cadre naturel et ouvert à tous, traduit le caractère transparent et donc légitime de l'autorité royale finalement acquise par Arthur : ce n'est pas par la guerre ni par des tractations politiques que le personnage accède au pouvoir, mais par une épée merveilleuse dont il s'empare aux yeux de tous. Le décor constitue ainsi un moyen d'interprétation de la légitimité politique du possesseur d'Excalibur.

La présentation du décor et la question du caractère naturel du paysage soulignent parfois le rôle ambivalent du personnage de Merlin, qui intervient pour permettre à Arthur d'accéder à l'épée. En effet, cette figure d'enchanteur est traditionnellement considérée comme étant proche de la nature. Cependant, Merlin manipule également le pouvoir, et joue un rôle politique essentiel dans l'accession d'Arthur au trône. Cette intervention décisive du personnage est d'ailleurs suggérée dès le roman de Robert de Boron, où l'enchanteur annonce ce moyen d'élection divine (Robert de Boron, 264). Sans remettre totalement en question la légitimité du pouvoir arthurien, les séries Merlin et Camelot soulignent toutefois ce rôle de Merlin dans l'orchestration des éléments naturels permettant au jeune Arthur de s'emparer de l'épée. Dans la série britannique Merlin, c'est le sorcier lui-même qui plante Excalibur dans un rocher au milieu de la forêt (S03E13, "The Coming of Arthur, Part $2 »)$. Le prodige est représenté dans sa matérialité, en insistant sur le geste magique de Merlin, lequel fait le choix délibéré d'intégrer Excalibur à un espace reculé et naturel. De même, c'est ensuite lui qui conduit Arthur à ce rocher, afin qu'il puisse retirer l'épée aux yeux du peuple et des chevaliers rassemblés (S04E13, "The Sword in the Stone, Part $2 »)$. Par sa magie, Merlin donne la possibilité à Arthur de libérer la lame : il permet ainsi au peuple d'être témoin du statut supérieur du jeune roi. Excalibur revêt ici une fonction de placebo, en devenant le symbole d'une légende forgée de toute pièce par Merlin, et entièrement orchestrée par l'enchanteur. Une perspective similaire est développée dans Camelot, où Merlin apparaît comme une figure dissimulatrice, si ce n'est manipulatrice. Dans la série, l'épée de Mars comme Excalibur servent à consolider l'autorité du jeune Arthur, et chaque fois l'accession du roi à l'épée est organisée par Merlin. L'épée de Mars, plantée en haut d'une cascade, entraîne la 
démonstration d'une prouesse physique d'Arthur et lui permet de se distinguer comme étant le héros annoncé par la prophétie de l'épée (S01E02, "The Sword and the Crown »). Or, la série suggère que l'épée a été placée là par Merlin lui-même, par anticipation, afin de préparer l'avènement d'Arthur. De même, la mise en scène d'Excalibur, quelques épisodes plus tard, montre que la légende est entièrement forgée par Merlin (S01E04, "Lady of the Lake »). Dès lors, le pouvoir d'Arthur et sa légitimité sont discrètement remis en question, puisque l'intégration de l'épée à un paysage naturel correspond en réalité à une démarche artificielle, née de la manipulation politique de l'enchanteur.

\section{S’approprier la puissance d'une épée légendaire}

Présenter Excalibur dans un cadre naturel ou à travers des principes artificiels permet d'éclairer la nature du pouvoir attribué au porteur de l'épée. Alors que les séries arthuriennes valorisent la présence du décor naturel, les séries qui ne proposent qu'une référence ponctuelle à Excalibur tendent à l'intégrer à des espaces clos. L'épée apparaît alors dans des scènes intérieures, qui réduisent tout accès au paysage.

\section{La fermeture du décor dans les cadres sériels non-arthuriens}

17 En dehors des représentations arthuriennes, l'épée est conçue comme un trésor caché, appartenant à des collections privées et secrètes. C'est dans cette logique qu'elle est présentée dans la série Da Vinci's Demons (Starz, 2013-2015) où le héros, Léonard de Vinci, est invité à parcourir les sous-sols du Vatican, qui regorgent de trésors et d'objets merveilleux (S01E07, «The Hierophant ») : un crâne de dragon, la lance de Longin, un masque de pharaon, etc. Ces éléments ne sont pas tous décrits en détail. Parmi ceux-ci, il est possible de distinguer une épée plantée jusqu'à la garde dans la roche : si cette arme n'est pas nommée, la présence de la pierre la rend toutefois clairement identifiable. Le mythe d'Excalibur est ici déplacé dans le temps et dans l'espace, afin d'intégrer une collection d'objets rares et précieux, employés pour impressionner le héros. De façon similaire, la série animée The Simpsons (FOX, 1989- ) laisse à son tour apparaître brièvement Excalibur, qui rejoint les antiquités et les trésors conservés dans le sous-sol du riche Mr. Burns (S05E04, «Rosebud»). La série présente une grande accumulation d'objets, qui défilent en arrière plan et au premier plan tandis que Mr. Burns et Mr. Smithers, son assistant, se déplacent au cœur de cette collection. Les objets encadrent ainsi le chemin des deux personnages. Parmi ces trésors, il est possible de distinguer plusieurs momies et sarcophages, la Vénus de Milo, les Tables de la Loi, ainsi qu'Excalibur, nommée et identifiée par Mr. Smithers. Ces collections prestigieuses concentrent de nombreux attributs du pouvoir et de la richesse, et accordent ainsi une place de choix à l'épée du roi Arthur.

Excalibur est alors plus ou moins valorisée pour elle-même. En effet, elle n'est que mentionnée en passant, et n'apparaît que brièvement dans The Simpsons, où elle contribue à l'effet d'accumulation du trésor entreposé par Mr. Burns. Il est à noter qu'il s'agit de l'unique représentation sérielle qui présente l'épée plantée dans une enclume, et non dans un rocher. Si Excalibur apparaît plus longuement dans Da Vinci's Demons, elle n'y est toutefois pas nommée, et n'est pas même mentionnée par son propriétaire, qui privilégie un autre de ses trésors. À l'inverse, l'épée plantée dans un rocher occupe une salle à part entière dans la série Supernatural (The WB et The CW, 2005-présent), où elle est conservée 
dans le sous-sol d'une riche médiéviste (S06E12, "Like a Virgin »). Ici, Excalibur est décrite comme une épée merveilleuse parmi d'autres, forgée dans le sang d'un dragon. Bien que Supernatural ne fasse pas apparaître à l'écran l'épée identifiée comme Excalibur, l'autre lame présentée reprend toutefois les mêmes codes esthétiques et symboliques : il s'agit d'une épée plantée en diagonale dans un rocher, et mise en valeur pour sa puissance. Si la médiéviste possède de nombreux autres objets précieux placés en exposition, cette arme possède toutefois un statut à part et est conservée avec soin dans son rocher, transporté au milieu d'une salle gardée secrète. Ces éléments permettent un renvoi direct à l'épée du roi Arthur. Le héros, Dean Winchester, demande d'ailleurs en découvrant cette arme s'il s'agit d'Excalibur. Cependant, malgré cette apparente valorisation dans l'épisode, l'épée ne constitue pas une fin en soi mais reste avant tout un outil, employé pour combattre un dragon. La représentation de l'épée n'est ainsi pas nécessairement liée à son importance dans la narration. Dans la série The Librarians (TNT, 2014-2015), Excalibur est une épée enchantée, libérée de son rocher et qui possède une volonté propre (S01E01, "And the Crown of King Arthur »). Cette épée, qui vole et se déplace à sa guise, garde l'entrée d'une bibliothèque magique qui renferme les connaissances merveilleuses du monde. Excalibur n'est alors pas valorisée pour ellemême, mais constitue un objet mythique parmi d'autres. Séparée du cadre restreint de sa légende, l'épée devient un symbole plus vaste de pouvoir.

Dans ces interprétations, l'épée apparaît dans des espaces clos et sombres, bien éloignés des vastes paysages présentés dans les séries arthuriennes. Excalibur est intégrée à des pièces dissimulées, jalousement gardées dans des espaces souterrains auxquels on accède le plus souvent par un escalier. Dans Da Vinci's Demons, le héros doit même emprunter une plateforme secrète pour pouvoir accéder à la salle des trésors. Dans Once Upon a Time, la deuxième apparition de l'épée dans le rocher insiste sur cette dissimulation : si, dans un premier temps, Excalibur présentée dans un cadre naturel revient bien au roi Arthur, la série joue toutefois sur la représentation parallèle de différentes temporalités et l'épée reparaît par la suite, de nouveau dans son rocher, non plus dans le Moyen Âge arthurien mais dans la ville de Storybrooke au XXI e siècle (S05E02, "The Price »). L'épée est alors conservée par un personnage considéré à ce moment de l'intrigue comme un méchant, qui garde Excalibur et le rocher dans son sous-sol, derrière une porte lourdement verrouillée. Cet espace clos et souterrain prend l'apparence d'une grotte et n'est que peu éclairé. Celle qui possède alors l'épée, Emma Swan, cherche de fait à dissimuler sa puissance aux autres personnages. Privilégiant une autre approche, Supernatural justifie cet emplacement souterrain et secret en insistant sur son rôle de protection. De même, le socle rocheux y est décrit comme un moyen de préserver l'épée. Si la pierre est conservée, elle perd toutefois son lien avec l'espace naturel. Excalibur, toujours plantée dans son rocher, semble avoir été déplacée et mise à l'écart. Or, dans ce type de représentation non-arthurienne, le déplacement ne sert pas tant la protection de l'épée que l'appropriation de son pouvoir par des personnages sans aucune légitimité.

\section{Le déplacement de l'épée comme détournement du pouvoir}

En étant tirée de son cadre extérieur, Excalibur est dissimulée au monde. La disparition des éléments naturels du décor renvoie dès lors à la disparition de la légitimité vis-à-vis de la puissance ainsi acquise : l'épée est déplacée pour être réservée à un seul, qui n'est pas apte à l'extraire de son rocher. En effet, dans ces mises en scène, Excalibur apparaît chaque fois dans la pierre ou dans l'enclume, suggérant que l'épée ne peut être déplacée 
qu'avec son socle, et que nul ne peut l'en tirer. L'épée et la pierre continuent d'apparaître de façon conjointe, dans des lieux sombres qui contrastent avec la présentation d'Excalibur aux yeux de tous dans les séries arthuriennes - ainsi qu'au cinéma. Par exemple, Da Vinci's Demons et Once Upon a Time prennent pour cadre un sous-sol creusé dans la pierre. L'épée semble ici dissimulée au fond d'une grotte qui demeure toutefois un espace artificiel creusé par la main de l'homme - ou, dans le cas de Once Upon a Time, par magie. L'espace naturel et souterrain est exploité et mis au service de l'action humaine, à des fins d'appropriation du pouvoir d'Excalibur. Le lieu n'est alors éclairé que par de minces puits de lumière, qui impliquent l'utilisation par les personnages de torches enflammées.

21 L'épée et son rocher sont ainsi dissimulés par des figures autres que le souverain légitime, posant la question du véritable possesseur d'Excalibur. Le fait que l'arme demeure entreposée dans un espace clos, sans bouger de la pierre, indique que son possesseur ne peut l'en extraire. Dès lors, qui est apte à accéder à Excalibur ? Beaucoup de prétendants essayent, en particulier dans les séries consacrées au roi Arthur : l'échec de nombreux candidats constitue alors un moyen de valoriser Arthur, qui seul parvient à accomplir ce prodige. Ainsi, dans Once Upon a Time comme dans Kaamelott, plusieurs personnages échouent à extraire l'épée, dont ils ne souhaitent que s'approprier la puissance. Leur prétention au pouvoir n'est pas légitime, voire n'est pas honnête, et leur ambition est alors mise à mal par la magie de l'épée.

D'autres séries détournent parfois la figure du roi et n'accordent pas l'extraction de l'épée à Arthur. Ainsi, dans Galavant, c'est le roi Richard qui, accédant enfin au statut de héros, affirme son autorité royale en retirant d'une souche l'épée légendaire, au-dessus de l'inscription suivante: "The Hero Sword To Be Drawn by The One True King " (S02E02, «World's Best Kiss »). Dans Da Vinci's Demons, le héros approche ses mains pour saisir la garde de l'épée, mais n'achève jamais son geste : son attention est appelée ailleurs, si bien que la révélation d'une possible puissance supérieure du personnage est laissée en suspens ${ }^{3}$. Excalibur demeure associée aux héros. La série Supernatural joue sur l'attente du spectateur de voir Dean Winchester réussir ce test. Lorsqu'il approche de l'épée pour s'en saisir, la musique épique résonne, mais s'achève dans un renversement héroïcomique. En effet, inapte à extraire l'épée de son socle de pierre, le « héros » applique des techniques modernes à cette tradition légendaire : employant des explosifs pour détruire le rocher, il finit par briser la lame de l'épée ${ }^{4}$. La série affiche ici la difficile confrontation du mythe médiéval avec la technologie destructrice du XXI ${ }^{e}$ siècle. Le héros n'est pas roi, ni même chevalier, mais il constitue le seul «héros » possible dans la narration: l'épée brisée devient le signe de nouvelles ambitions, puisque les personnages doivent alors se contenter d'une lame brisée, brandie par un héros loin de la dignité royale.

Le paysage naturel entourant Excalibur, inventé dans les productions audiovisuelles du $\mathrm{XX}^{\mathrm{e}}$ siècle, apparait par conséquent lié à la présence d'un porteur légitime. À l'inverse, le décor intérieur et artificiel, chaque fois sombre et souterrain, et uniquement exploité par les séries télévisées non-arthuriennes, devient le signe d'une tentative de détournement du pouvoir - royal ou merveilleux - conféré à l'épée. Le déplacement d'Excalibur, qui n'est plus laissée à la vue de tous mais est jalousement gardée et enserrée dans son rocher, souligne l'absence d'harmonie entre l'arme et son possesseur, qui ne présente aucune légitimité vis-à-vis de la puissance d'Excalibur. Les créations sérielles développent ainsi une toute nouvelle ligne narrative pour l'épée légendaire, en la déplaçant de son cadre originel et en suggérant une volonté de détournement de son pouvoir. Pour le 
cinéma et la télévision, qui effacent l'interprétation chrétienne privilégiée par la littérature médiévale, Excalibur doit apparaitre dans un paysage ouvert, accessible à tous. Les séries télévisées amplifient cette iconographie née au cinéma en associant le décor aux questions de légitimité. Par conséquent, si le roi Arthur n'est pas mis en scène, la place de l'épée doit certes être dans son rocher, mais également dans un espace naturel plus vaste où l'eau, la terre et la végétation s'unissent pour valoriser cet instrument de pouvoir. Le décor joue ainsi un rôle essentiel dans la construction de l'intrigue, en annonçant la nature de la puissance d'Excalibur, ainsi que la légitimité de son possesseur. Loin de se limiter à une fonction illustrative, le paysage entre en résonnance avec la narration, et reflète les enjeux diégétiques développés dans les différentes séries.

\section{Sériographie}

« And the Crown of King Arthur. » Réal. Dean Devlin. The Librarians, S01E01. TNT. 7 décembre 2014.

« Lady of the Lake. » Réal. Jeremy Podeswa. Camelot, S01E04. Starz. 15 avril 2011.

« La Promesse. » Réal. Alexandre Astier. Kaamelott, S05E22. M6. Mai 2007.

« La Roche et le Fer. » Réal. Alexandre Astier. Kaamelott, S05E12. M6. Mai 2007.

« Like a Virgin. » Réal. Phil Sgriccia. Supernatural, S06E12. The CW. 4 février 2011.

« Rosebud. » Réal. Wes Archer. The Simpsons, S05E04. FOX. 21 octobre 1993.

« The Coming of Arthur, Part 2. » Réal. Alice Troughton. Merlin, S03E13. BBC One. 4 décembre 2010.

« The Dark Swan. » Réal. Ron Underwood. Once Upon a Time, S05E01. ABC. 27 septembre 2015.

« The Hierophant. » Réal. Michael J. Basset. Da Vinci’s Demons, S01E07. Starz. 31 mai 2013.

« The Price. » Réal. Romeo Tirone. Once Upon a Time, S05E02. ABC. 4 octobre 2015.

« The Sword and the Crown. » Réal. Ciaran Donnelly. Camelot, S01E02. Starz. 1 ${ }^{\mathrm{e}}$ avril 2011.

« The Sword in the Stone, Part 2. » Réal. Alice Troughton. Merlin, S04E13. BBC One. 24 décembre 2011.

« World's Best Kiss. » Réal. John Fortenberry. Galavant, S02E02. ABC. 3 janvier 2016.

\section{Bibliographie}

Robert de Boron. Merlin, Roman du XIII siècle. Genève : Droz, 1979.

White, Terence Hanbury. The Once and Future King. New-York : Ace Books, 1996. 


\section{NOTES}

1. Il est possible de citer The Natural (1984) de Barry Levinson, Arthur et les Minimoys (2006) de Luc Besson, ainsi que Minions (2015) de Kyle Balda et Pierre Coffin. Ces rares occurrences font appel à une image modernisée d'Excalibur, et y consacrent un long temps narratif. Contrairement aux séries non-arthuriennes, les films non-arthuriens développent le rôle de l'épée, laquelle est intégrée à la narration et ne constitue pas un simple élément accessoire dans l'intrigue.

2. Dans l'épisode pilote, Excalibur apparaissait plantée dans un rocher situé au milieu d'un lac.

3. Il est à noter que l'apparition d'Excalibur est annoncée discrètement au début de l'épisode, lorsque Léonard de Vinci, faisant une pause dans son parcours, apparaît à côté de sa longue dague plantée dans le sol (02'18"). S'il ne s'agit pas d'Excalibur, et si le symbole n'est pas davantage exploité, l'image de la lame plantée verticalement près du héros demeure. De façon intéressante, la série ne présente pas le geste d'insertion de la dague dans le sol, mais affiche simplement le moment où Léonard l'en retire pour la remettre à sa ceinture.

4. L'épée brisée constitue également un motif essentiel de la série Once Upon a Time, où la pointe d'Excalibur est rompue, et permet de former une dague à part. La continuité entre ces deux armes est accentuée par la représentation de la même lame gravée et sinueuse - Once Upon a Time proposant le seul exemple audiovisuel d'une Excalibur ondulée, qui reprend quelque peu l'esthétique d'un poignard malais.

\section{AUTEUR}

\section{JUSTINE BRETON}

Justine Breton est Agrégée de Lettres modernes et doctorante en littérature médiévale à l'Université de Picardie Jules Verne (Amiens), elle étudie les représentations du pouvoir dans la légende arthurienne, dans une optique comparatiste mettant en relation les sources médiévales du mythe et leurs adaptations cinématographiques et sérielles. Ses travaux l'ont notamment amenée à analyser la dimension littéraire et esthétique de séries télévisées contemporaines, telles que Kaamelott ou Game of Thrones.Université de Picardie Jules Verne 